\title{
From The National Perinatal Information Center: Narrowing the Gap in Perinatal and Neonatal Disparities: The Race and Ethnicity Dashboard
}

Elizabeth Rochin, PhD, RN, NE-BC

The National Perinatal Information Center (NPIC) is driven by data, collaboration and research to strengthen, connect and empower our shared purpose of improving patient care.

For over 30 years, NPIC has worked with hospitals, public and private entities, patient safety organizations, insurers and researchers to collect and interpret the data that drives better outcomes for mothers and newborns.

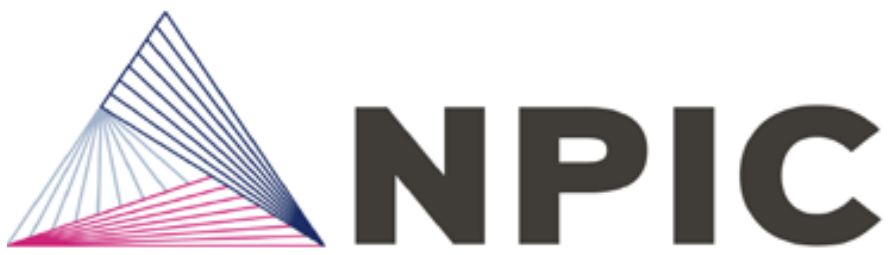

National Perinatal Information Center

"In June 2020, the National Perinatal Information Center presented information on the importance of race and ethnicity reporting, particularly surrounding perinatal and neonatal outcomes. To understand outcomes necessitates the understanding of the communities that are cared for and the potential gaps that exist in quality care."

In June 2020, the National Perinatal Information Center presented information on the importance of race and ethnicity reporting, particularly surrounding perinatal and neonatal outcomes. To understand outcomes necessitates the understanding of the communities that are cared for and the potential gaps that exist in quality care.

Racial and ethnic disparities continue to generate widespread interest and concern, particularly among maternal morbidity and mortality researchers. Maternal outcome disparities, particularly maternal mortality, warrant immediate attention and focus on assuring a standardized care approach, rigorous quality assessment, and rapid cycle improvement. The wide variation in obstetrical outcomes across hospitals, poor overall performance on perinatal indicators, and the persistent racial and ethnic disparities in obstetric and perinatal outcomes require innovative remedies that tackle these challenges together(1). In 2019, expert obstetric disparities researcher Dr. Elizabeth Howell recommended the fol- lowing eight (8) steps(2) for narrowing gaps in maternal outcome disparities:

$\begin{array}{ll}\checkmark & \text { Enhance team communication } \\ \checkmark & \text { Address implicit bias } \\ \checkmark & \text { Implement a race and disparities dashboard } \\ \checkmark & \text { Perform enhanced severe maternal morbidity and severe } \\ & \text { maternal mortality reviews } \\ \checkmark & \text { Standardize care in Perinatal settings } \\ \checkmark & \text { Promote a culture of equity } \\ \checkmark & \text { Develop new models for Respectful Care } \\ \checkmark & \text { Engage key stakeholders (internal and external) }\end{array}$

\section{"The efforts of utilizing a race and disparities dashboard illustrate hospital or system overall results and stratify these measures by race and ethnicity to understand and quantify the disparities that exist locally within a hospital or system."}

The efforts of utilizing a race and disparities dashboard illustrate hospital or system overall results and stratify these measures by race and ethnicity to understand and quantify the disparities that exist locally within a hospital or system. Such dashboards are an important snapshot of performance, and the data contained therein can then be measured over time to monitor local quality improvement efforts(3). Dedicated and coordinated inpatient team efforts to reduce disparities, including documentation of race and ethnicity at the time of admission, must be a priority for those serving our communities. Earlier discussions included the importance of assuring self-reporting of race and ethnicity to enhance the quality and accuracy of that reporting.

With as few as $14 \%$ of healthcare organizations using patient data to assess variation in care and outcomes(4), it is imperative that race and ethnicity reporting be incorporated in all quality improvement projects and those whose voices are most likely to be impacted involved in the development and execution of those improvement programs.

In November 2020, the National Perinatal Information Center deployed a Race and Ethnicity Dashboard with all quarterly reporting, with both maternal and newborn outcomes, a routine component of the quarterly data package received by hospitals going forward. The goal of this reporting is to serve as an adjunct to diversity and inclusion programs, as well as to serve as a catalyst for discussion related to quality improvement/patient experience initiatives within racial and diversity lenses. 
National Perinatal Information Center $4 / 01 / 2019-3 / 31 / 2021$

Total Deliveries: $n=\mathbf{2 9 0 , 2 8 8}$

Rates as a Percent of Discharges withineach Race/Ethnicity Category

Cesarean Section

NPIC DB $=33.9 \%$

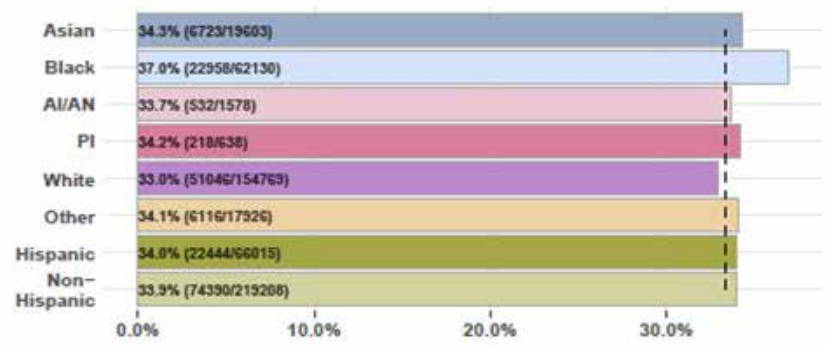

National Perinatal Information Center $4 / 01 / 2019-3 / 31 / 2021$

Total Deliveries: $n=\mathbf{2 9 0 , 2 8 8}$

Rates as a Percent of Discharges withineach Race/Ethnicity Category

Severe Maternal Morbidity ${ }^{1}$ (SMM): Overall

NPIC DB $=2.2 \%$

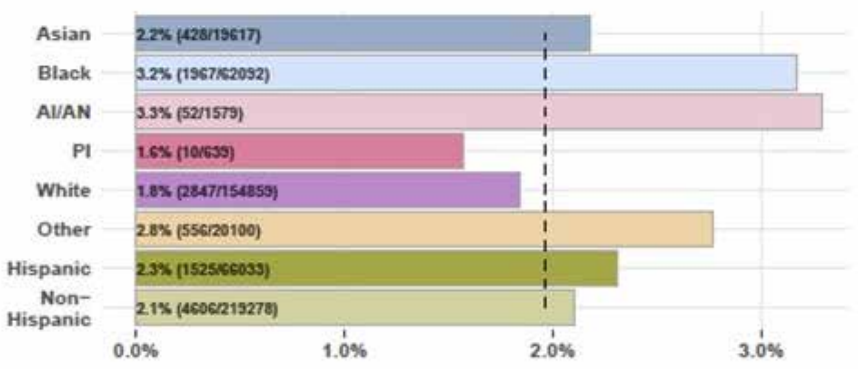

National Perinatal Information Center $4 / 01 / 2019-3 / 31 / 2021$

Total Neonates: $n=303.095$

Rates as a Percent of Discharges withineach Race/Ethnicity Category

Special Care Discharges ( $\%$ of Neonates)

NPIC DB $=15.9 \%$

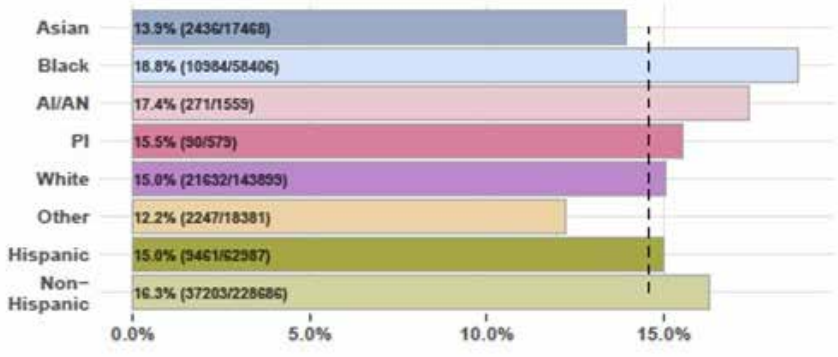

The penultimate goal of quality improvement initiatives in obstetric, perinatal and neonatal programs is the elimination of preventable maternal and neonatal morbidity and mortality. Understanding the issues of systemic racism and implicit bias on outcomes must be an integral component of any patient safety program. Coupling race and ethnicity data and patient outcomes creates a much more robust and transparent vantage point of where vulnerabilities exist in patient care.
"Understanding the issues of systemic racism and implicit bias on outcomes must be an integral component of any patient safety program. Coupling race and ethnicity data and patient outcomes creates a much more robust and transparent vantage point of where vulnerabilities exist in patient care."

\section{References:}

1. Howell, E. A., \& Zeitlin, J. (2017). Quality of Care and Disparities in Obstetrics. Obstetrics and Gynecology Clinics of North America, 44(1), 13-25. https://doi.org/10.1016/j. ogc.2016.10.002.

2. Howell, E. A., \& Ahmed, Z. N. (2019). Eight steps for narrowing the maternal health disparity gap: Step-by-step plan to reduce racial and ethnic disparities in care. Contemporary Ob/Gyn, 64(1), 30-36.

3. Howell, E.A., Brown, H...Grobman, W.A. (2018). Reduction of peripartum racial and ethnic disparities: A conceptual framework and maternal safety consensus bundle. JOGNN, 47, 275-289.

4. MGH Disparities Solutions. (2019). Disparities solutions center. https://mghdisparitiessolutions.org/

The author has no conflicts of interests to disclose.

\section{NT}

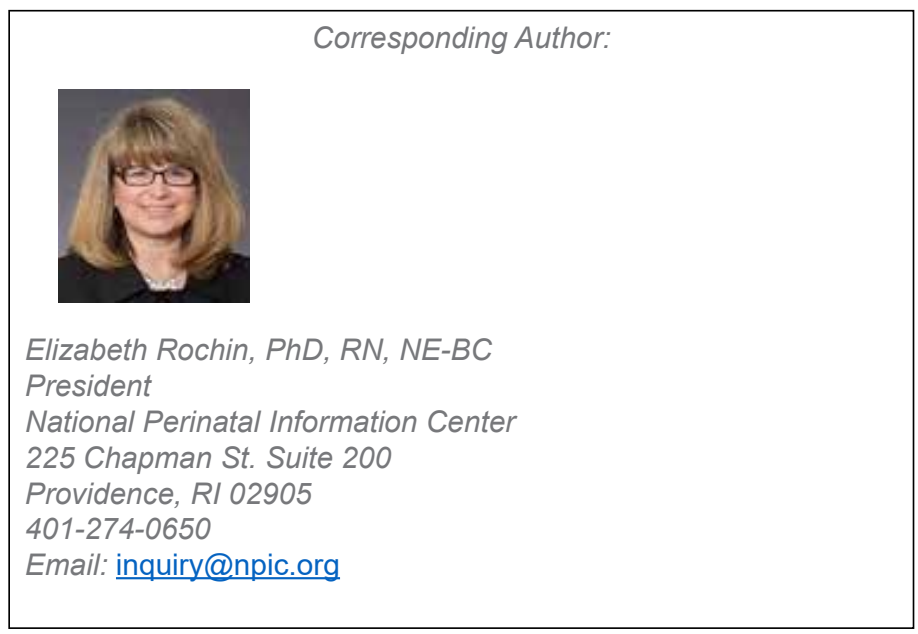

